\title{
Nasopharyngeal Carcinoma
}

National Cancer Institute

\section{Source}

National Cancer Institute. Nasopharyngeal Carcinoma. NCI Thesaurus. Code C3871.

A carcinoma arising from the nasopharyngeal epithelium. It includes the following types:

keratinizing squamous cell carcinoma, nonkeratinizing carcinoma (differentiated and

undifferentiated), basaloid squamous cell carcinoma, and papillary adenocarcinoma. 